\title{
MAN_01 - Process management implementation for RT-qPCR activities in Bio-Manguinhos research and development sector
}

Juliana Fernandes Amorim da Silva ${ }^{1 *}$; Renata Tourinho Cantinho Brício ${ }^{1}$; Aloysio Moreira Junior ${ }^{1}$; Hedione Soares Müler ${ }^{1}$; Gisela Freitas Trindade ${ }^{1}$; Sheila Maria Barbosa de Lima ${ }^{1}$.

${ }^{1}$ Fiocruz/Bio-Manguinhos.

Introduction: The current technological investments in public health and increasing demand for diseases diagnosis, treatment and prevention, form a scenario where reducing time and costs is essential to develop and produce immunobiologicals to control infectious diseases outbreaks and epidemics.Research and Development (R\&D) is the pharmaceutical key sector in which product quality must be follow up and assured before product reaches the manufacturing stage or customer. In this respect, it is extremely important to concentrate efforts in the R\&D activities process management to control and improve its processes.

Objective: Thus, the present work aimed to report the processes management in Bio-Manguinhos R\&D department. The case study was carried out at the Virological Technology Laboratory (LATEV - BioManguinhos), for real-time PCR technique (RT-qPCR), in which the process management implementation is ongoing.

Methodology: The current operational processes were defined and designed using the process mapping methodology and analyzed under a perspective of continuous improvement, based on the PDCA cycle through quality tools. The ishkawa diagram helped to identify a link between the main causes of highlighted issues for the current PCR technique process and their respective improvement opportunities. Then, an action plan for optimization process was proposed using $5 \mathrm{~W} 2 \mathrm{H}$ tool and it was redesigned applying the flowchart tool. New RT-qPCR methodology was validated using an adapted protocol based on a set of national and international normative instructions.

Results: This study demonstrated that the method and tools chosen to optimize the RT-qPCR technique processes were effective, as expected. Using the Lean thinking philosophy, improvement opportunities were identified, which made the processes more fit, less costly, time-consuming and laborious. In addition, it was possible to upgrade data tracking and integrity. The new RT-qPCR methodology has been successfully validated by statistical analysis, to quantify the Zika Virus, making the new method reliable, safe, auditable and with quality.

Conclusion: Thus, process management has facilitated the LATEV molecular biology team to visualize operational constraints, resulting in an increasing RT-qPCR test throughput and, consequently, internal and external client service. This provided the ability to carry out a continuously critical analysis of the RT-qPCR processes for future improvements. Bio-Manguinhos also has a significant gain in relation to the value chain of the RT-qPCR service, offered with quality and client focus. In addition, there is an optimization of public health investments, attending and benefitting Brazilian society.

Keywords: process management; optimization process; real-time PCR 